\title{
(Re)pensando nuestra democracia
}

\author{
Juan Pablo Pozo Bahamonde* \\ https://doi.org/10.35242/RDE 2020302
}

\section{Nota del Consejo Editorial}

Recepción: 31 de mayo de 2020.

Revisión, corrección y aprobación: 17 de junio de 2020.

Resumen: El régimen democrático contemporáneo, el Estado de derecho y la celebración de elecciones periódicas en América Latina y el Caribe conmemoran 40 años de vigencia en la región, afrontando los retos derivados de las limitaciones de la democracia formal y representativa; y de su acción en generar mejores condiciones de vida e inclusión a amplios sectores de la población como una real materialización de los derechos humanos. En este contexto, el presente artículo procura ofrecer un análisis de los retos y desafíos de la democracia latinoamericana y caribeña en la segunda década del siglo XXI, y establece cuáles serían los ejes de acción para fortalecer la triada de derechos, garantías e institucionalidad democrática a través de la construcción de una identidad democrática propia, el fortalecimiento de la institucionalidad publica, el diseño de políticas públicas con participación ciudadana y la incorporación del enfoque de protección de derechos humanos en los ámbitos político-electorales.

Palabras clave: Democracia / Debilitamiento de la democracia / Derechos humanos / Elecciones / Reformas democráticas / Políticas públicas / Institucionalidad pública.

Abstract: The contemporary democratic regime, the State of Law and periodic electoral processes in Latin America and the Caribbean celebrate 40 years in the region facing the challenges that arise from the constraints of formal and representative democracy and its action in generating better living conditions and inclusion of broad sectors of the population as a real materialization of human rights. Within this context, this article provides an analysis of the challenges of the Caribbean and Latin American democracy in the second decade of the $21^{\text {st }}$ century. It also establishes the roadmap to strengthen the triad of rights, guarantees and democratic institutionality through the construction of a democratic identity, the strengthening of public institutionality, the design of public policies with citizen participation, and the incorporation of the human rights protection focus in the political and electoral scopes.

Key Words: Democracy / Weakening of democracy / Human rights / Elections / Democratic reforms / Public policies / Public institutionality.

\footnotetext{
* Ecuatoriano, abogado, correo jppozob@hotmail.com. Especialista de la Secretaría para el Fortalecimiento de la Democracia de la Organización de Estados Americanos. Cursa un doctorado en Estado de Derecho y Gobernanza Global por la Universidad de Salamanca, España (2020). Doctor en jurisprudencia y abogado por la Universidad de Cuenca, Ecuador (2002). Mediador por la Universidad de Buenos Aires, Argentina (2005); especialista en Métodos Alternativos de Solución de Conflictos Sociales por la Universidad Andina Simón Bolívar, Quito, Ecuador (2012). Magíster en Derecho del Mercado por la Universidad Andina Simón Bolívar, Quito, Ecuador (2016). Consultor y observador electoral, articulista, docente universitario. Exconsejero del Consejo Nacional Electoral del Ecuador (2011-2015). Expresidente de la Función Electoral y del Consejo Nacional Electoral del Ecuador (2015-2017). Autor del libro "Democracia en el Contexto Suramericano" (2015).
} 


\section{DERECHOELECTORAL}

\section{El RÉgimen democrático CONTEMPORÁneo de AmÉrica latina y el CARIBE: RETOS Y PERSPECTIVAS}

Entre los años 2018 y 2020 se marca un hito histórico transcendental para América Latina y el Caribe, al celebrarse 40 años de vigencia del sistema democrático, la prevalencia de los derechos humanos, la celebración de elecciones periódicas y la vigencia del Estado de derecho en la región.

Sin duda, nunca antes en la historia independiente de los Estados latinoamericanos y caribeños el sistema democrático ha prevalecido y ha estado incólume durante tanto tiempo. Una evidencia clara de esta aseveración es el ejercicio continuo de los ciudadanos de su derecho al voto en elecciones periódicas, libres y competitivas, marcadas por el pluralismo político, la prevalencia de la representatividad democrática y la alternancia política.

Así, entre los años 1979 y 2019 se han producido cinco grandes ciclos electorales en los países de la región: i) 1989, ii) 1994, iii) 2015-2006, iv) 2013-2014 y v) 2017-2019, en los cuales como lo indica Flavia Freidenberg:

(...) un número significativo de países tuvieron elecciones presidenciales, legislativas $\mathrm{y} / \mathrm{o}$ municipales en el mismo momento, lo que da cuenta de ciclos de elecciones que ponen en evidencia la salud de las democracias latinoamericanas. Dado que las elecciones son el corazón de la democracia representativa, el hecho de que se celebren elecciones libres, justas y competitivas facilita que la ciudadanía viva en un sistema democrático. (2014, p. 268).

Ahora bien, esta hegemonía de la democracia representativa y electoral en la región durante estos 40 años ha permitido enraizar a la democracia como un anhelado sistema de gobierno conjuntamente con la defensa de sus valores fundamentales: soberanía popular ejercida mediante el voto, elecciones periódicas, libres y justas, pluralidad política, separación e independencia de los poderes públicos.

Por otro lado, este contexto ha contribuido a establecer una «institucionalidad formal» en la cual el Estado, su ordenamiento jurídico y estructura orgánica deben garantizar un real ejercicio de los derechos humanos y procesar las crecientes demandas sociales. En este sentido, la 


\section{DERECHO EIECTORAL}

tríada de derechos, garantías e institucionalidad estatal coopera para construir una real sociedad democrática en beneficio de sus ciudadanos.

Sin embargo, en la práctica este «deber ser» de la democracia formal en nuestra región aún constituye una tarea pendiente de alcanzar, así como sigue siendo un deseo la mejora de los niveles de vida de los ciudadanos dentro de una sociedad democrática. Como lo refiere Dieter Nohlen:

(...) Uno de los mayores desafíos consiste en combatir con éxito la pobreza y promover, ahora ya, la justicia social. Este desafío no sólo es un objetivo en sí mismo, sino también una condición necesaria para que la propia democracia tenga futuro en la región. Esta tesis es una convicción ampliamente compartida en América Latina, incluso por los gobiernos de turno (...). (2003, p. 41).

Los indicadores de la Comisión Económica para América Latina de las Naciones Unidad (CEPAL) evidencian este déficit o deuda de nuestros sistemas democráticos, por ejemplo: "(...) en 2019 la pobreza aumentó en un $29,8 \%$ en la región, significando un aumento de 27 millones de personas pobres con respecto al año 2014" (CEPAL, 2019, p. 18).

Por otro lado, América Latina sigue siendo la región más desigual del mundo en materia de ingresos de su población; esto es un obstáculo al desarrollo y a la garantía de los derechos y del bienestar de las personas. La desigualdad extrema reproduce privilegios, erosiona la democracia, corrompe la política y genera pobreza. Al parecer siguen teniendo vigencia las palabras del expresidente de Chile, Salvador Allende, quien en 1971 durante su visita oficial a Ecuador expresó:

(...) América Latina no puede seguir siendo el continente de la esperanza frustrada: América Latina no debe ser el continente potencialmente rico cuyos habitantes en un porcentaje tan alto saben del hambre, de la desocupación, de la falta de vivienda, de agua, de luz. América Latina ha dado ya demasiado para recibir tan poco (...). (Allende, 1971, párr. 3).

De los distintos informes sobre democracia, podemos destacar que la democracia continúa ampliando su alcance en todo el mundo, más de cuatro billones de personas viven actualmente en alguna forma de democracia; sin embargo, existe una profunda erosión democrática caracterizada por la pérdida de calidad democrática y las dificultades para cumplir las expectativas de los ciudadanos. Hoy en día se registran los 


\section{DERECHO ELECTORAL}

niveles más altos de desigualdad socioeconómica del mundo, lo que se ha traducido en un acceso muy desigual al poder político. Las tasas de delincuencia y violencia en América Latina y el Caribe son las más elevadas del mundo, esto sumado al alto grado de corrupción socava la confianza en la democracia y alimenta el descontento cívico.

En materia de participación electoral e igualdad política de género, somos la región que mayor participación electoral registra en comparación con el resto del mundo y la que más ha avanzado en materia de igualdad política de género en las últimas décadas.

Las organizaciones políticas están sufriendo una crisis de representación, que se deriva de su dificultad para adaptarse a la transformación social.

Así, esta esperanza frustrada ha producido una creciente erosión del sistema democrático, el Estado de derecho y la confianza de la ciudadanía hacia su institucionalidad y procedimientos.

Lo anterior coincide con lo apuntado por la Corporación Latinobarómetro en su último informe del año 2018, sobre un estudio de opinión realizado en 18 países de Latinoamérica, en el cual se analiza el apoyo a la democracia. Las encuestas de opinión pública muestran una caída de 12 puntos en el apoyo a la democracia durante ¿los últimos diez años?, del $60 \%$ en 2008 al $48 \%$ en 2017 , y un descenso cercano a los nueve puntos solo en los últimos tres años (2015-2017). En promedio, apenas el $16 \%$ de los entrevistados cree que la distribución de la riqueza es justa, el 32\% cree en su gobierno, el $24 \%$ confía en el sistema judicial, apenas el $21 \%$ en su Parlamento, el $28 \%$ en los órganos electorales y el $65 \%$ considera que la corrupción es un problema grave.

Definitivamente el apoyo a la democracia declina de manera sistemática año a año, el diagnóstico es que la democracia está enferma y corre el riesgo de hacerse crónica, los ciudadanos de la región están abandonado su apoyo al régimen democrático y prefieren ser indiferentes al tipo de régimen, por lo que se alejan de la política, la democracia y sus instituciones. Como lo señala Dieter Nohlen:

(...) El reciente desarrollo de la democracia en América Latina ha estado marcado por una creciente brecha entre la preferencia que este sistema político alcanza en la opinión pública y la confianza que los ciudadanos confiesan tener en las instituciones políticas. Mientras que el apoyo al ideario 


\section{DERECHO EIECTORAL}

democrático sigue alto, la satisfacción que la gente tiene con el funcionamiento de la democracia está en su punto más bajo desde la democratización (...). (2003, p. 41).

Mas allá de los indicadores, una muestra clara de esta erosión de la formalidad democrática puede verse en el último ciclo electoral de la región entre 2017 y 2019. En este, luego de la celebración de 15 elecciones presidenciales y legislativas en los países de la región, a continuación estalló en el segundo semestre del 2019 la denominada «primavera latinoamericana», caracterizada por amplios procesos de movilización y protesta social en Ecuador, Colombia, Perú, Argentina, Haití, Nicaragua, Chile, Bolivia, Brasil y Venezuela.

En todos ellos, en medio de una incertidumbre económico-social generalizada, más una aguda inestabilidad político-institucional, la mayoría de la ciudadanía reclamó una mayor participación en los asuntos públicos no solo desde el sufragio, sino que también exigió mejores condiciones de vida, la real vigencia de los derechos humanos, cuestionó la acción de sus gobernantes; y exigió al modelo político y económico vigente respuestas para solucionar sus problemas de subsistencia, salud, educación, vivienda, transporte, un real combate a la corrupción y la falta de inclusión real de las personas más vulnerables. Como lo refiere Francisco Guerrero:

Hemos sido testigos de un otoño turbulento, lleno de movilizaciones y protestas ciudadanas, cada vez más numerosas. Hong Kong, Quito, Barcelona, Santiago y La Paz. La gente abandona el silencio y la rabia contenida para expresar su molestia con el statu quo. El proceso de organización pasa necesariamente por las redes sociales. Las conciencias se sacuden haciendo temblar al establishment y la precaria gobernabilidad (...). (2019, párrs. 1-2).

Por otro lado, este agitado escenario regional durante 2019 vino acompañado de una tendencia global de reverdecimiento de ideas totalitarias, amenazas de guerra e intervención en países, nacionalismos a ultranza, xenofobia, lucha hegemónica entre naciones, liderazgos autoritarios y un populismo crónico, que amenazan a la democracia y a los derechos humanos. Como lo señala Sergio Bitar: 


\section{DERECHO EIECTORAL}

(...) La crisis de la democracia representativa puede desembocar en una regresión democrática (regímenes híbridos), o en una situación de anomia (vacío político y Estado fallido), u ocasionar la irrupción de gobiernos populista-autoritarios (...). (2020, párr. 7).

Dentro de esta preocupante tendencia global en la región, tenemos los datos presentados por el Índice de la Democracia 2019 realizado por la Unidad de Inteligencia del diario The Economist de Reino Unido, en el cual se concluyó que América Latina es la región más democrática entre las economías emergentes. Sin embargo, apenas 2 países ${ }^{1}$ son considerados «democracias plenas» con amplio pluralismo político-electoral y un adecuado funcionamiento del gobierno, alta participación política y respeto a los derechos civiles.

Por otro lado, según dicho índice, 13 países $^{2}$ de la región tienen «democracias imperfectas» en las cuales se reducen aspectos como el respeto a los derechos civiles, la participación política de la ciudadanía y el adecuado funcionamiento del gobierno. Se reconoce un pluralismo político-electoral de carácter formal.

En cuanto a los llamados «regímenes híbridos», 5 países ${ }^{3}$ latinoamericanos y caribeños entran en esta categoría, mientras 3 países de la región son situados como «regímenes autoritarios ${ }^{4} \gg$ en los cuales existe un marcado declive del pluralismo electoral-político y las libertades civiles.

Ante esto, parecería que estamos frente a un «eterno retorno» de las amenazas al sistema democrático de hace un siglo. Como está escrito en la historia, la Primera Guerra Mundial (1914-1918) provocó el colapso del Estado de derecho, el surgimiento del nazi-fascismo que desembocó en la Segunda Guerra Mundial (1939-1945). Sin embargo, a diferencia de aquella vez, nuestra región no escaparía de esas tendencias a causa de la desigualdad, la fragilidad económica y de la débil gobernabilidad al interior de nuestros países.

\footnotetext{
${ }^{1}$ Según el Índice de la Democracia 2019 únicamente Uruguay y Costa Rica son democracias plenas en América Latina y el Caribe.

${ }^{2}$ De acuerdo con el Índice de la Democracia 2019 son democracias imperfectas en Latinoamérica y el Caribe los siguientes países: Ecuador, Paraguay, México, Chile, Panamá, Argentina, Jamaica, Surinam, Brasil, Colombia, Guyana, Perú y República Dominicana.

3 El Índice de la Democracia 2019 caracteriza como regímenes híbridos a El Salvador, Bolivia, Honduras, Guatemala y Haití.

${ }^{4}$ El Índice de la Democracia 2019 caracteriza como regímenes autoritarios a Nicaragua, Cuba y Venezuela.
} 


\section{DERECHO EIECTORAL}

Al respecto, Dieter Nohlen claramente advierte:

(...) Por otra parte, se observan fenómenos que dan cuenta de enormes dificultades de las sociedades latinoamericanas para seguir gobernadas dentro del molde de una democracia representativa. No es que exista el peligro inminente de una involución, o sea de una sustitución de la democracia por un régimen autoritario como en tiempos anteriores. Lo que sí se observa son más bien fenómenos tendentes a desviar el ejercicio del poder de los padrones de la democracia representativa, tendencia incluso apoyada por el voto de los ciudadanos (...). (2003, p. 41).

A todo esto, el año $2020^{5}$ trajo consigo el desafío mundial de la pandemia del covid-19, provocando una crisis multidimensional sin precedentes que en pocos meses ha generado millones de contagiados y cientos de miles de fallecidos en los cinco continentes, que han desbordado las capacidades de los Estados y han creado enormes dificultades en todos los órdenes: sanitario, social, laboral, económico y democrático, lo que ha acrecentado la crisis y las brechas sociales existentes en varios países de la América Latina y el Caribe.

En el plano democrático, la pandemia del covid-19 ha generado la imposibilidad de celebrar las elecciones en las fechas previstas en sus marcos constitucionales y legales. Según el Instituto Internacional para la Democracia y Asistencia Electoral (IDEA), en su informe sobre el panorama global y mundial del impacto del covid-19 en las elecciones ${ }^{6}$, en un corto periodo comprendido entre febrero y mayo del 2020, al menos 52 países y territorios en todo el mundo han decidido posponer las elecciones nacionales y subnacionales. De estos, 10 países de América Latina han tenido que reagendar sus elecciones previstas para este año. Estos incluyen la reprogramación del plebiscito constitucional en Chile (del 26 de abril al 25 de octubre), el aplazamiento de las elecciones generales en Bolivia (nueva fecha por definir) y posponer elecciones locales en los estados de Hidalgo y Coahuila en México. Asimismo, las elecciones generales en República Dominicana fueron reprogramadas para el 5 de

\footnotetext{
${ }^{5}$ Según la Organización Mundial de la Salud (OMS), hasta el 29 de mayo América Latina y el Caribe registran un total de 800275 casos de COVID-19, donde los países más afectados con muertes y contagios son Brasil, Perú, Chile, México, Panamá, República Dominicana y Colombia.

6 IDEA (2020). Recuperado de https://www.idea.int/es/news-media/multimedia-reports/panorama-global-delimpacto-del-covid-19-en-las-elecciones
} 


\section{DERECHO EIECTORAL}

julio, y la segunda vuelta -si hubiera- para el 26 de julio. Otros países que se han visto en la necesidad de aplazar elecciones son Colombia, Perú, Argentina, Brasil, Uruguay y Paraguay.

A nivel mundial se tenía planificado la celebración de comicios en 86 países en diversas regiones, entre los que se destacan las elecciones presidenciales y legislativas de los países latinoamericanos señalados en el párrafo anterior, además de las elecciones en Estados Unidos y Venezuela.

Como lo señala Francisco Guerrero (2020):

Las secuelas de la pandemia, las altas posibilidades de contagio con la interacción humana, el riesgo de muerte, la aplicación de las medidas restrictivas antes mencionadas, ponen serias dificultades a la organización de los procesos electorales, por lo que deben analizarse alternativas democráticas para que no se vea lesionada la legitimidad de origen de los nuevos gobernantes, las transiciones democráticas, la alternancia de sus autoridades y la duración de los periodos de gobierno. Deben evitarse posibles prórrogas o ampliaciones de mandato de los gobiernos en ejercicio que contravengan los marcos internos constitucionales, hay que buscar alternativas en los mecanismos de votación que eviten vacíos de poder o acefalia de las instituciones, en momentos tan críticos y complejos en los que se debe enfrentar una pandemia (s.p).

Debemos estar atentos al gran peligro que representa el que aparezcan mimetizados en la mitad de la crisis la nefasta presencia del totalitarismo, el autoritarismo y el populismo, junto con la erosión de los derechos fundamentales, aprovechándose del desarrollo de los estados de excepción o emergencia.

Definitivamente, la democracia, los derechos y las libertades no pueden estar nunca en cuarentena; en este punto de quiebre histórico de la humanidad, es necesaria la activación de todos los mecanismos de participación y observación ciudadana para evitar el quebranto de los derechos fundamentales y el deterioro de la calidad de nuestras democracias regionales. La pandemia del covid-19 pone a prueba la resiliencia de los sistemas democráticos. 


\section{DERECHO EIECTORAL}

\section{2. ¿QUÉ HACER?-EJES DE ACCIÓN DEMOCRÁTICA}

Ahora bien, frente a este panorama regional complejo y mundial, es necesario plantear los aspectos especiales para fortalecer a la democracia, el Estado de derecho, las elecciones y los derechos humanos, a través de cuatro ejes fundamentales:

1) Construcción de una identidad democrática propia en América Latina y el Caribe.

2) Fortalecimiento de la institucionalidad democrática.

3) Diseño de políticas públicas con participación ciudadana que acompañen a los avances normativos.

4) Incorporación del enfoque de protección de derechos humanos en los ámbitos políticos-electorales.

Ahora bien, respecto al primer eje de la construcción de una identidad democrática propia en América Latina y el Caribe, nuestra región necesita ineludiblemente desarrollar un contenido teórico y doctrinario, propio y endógeno, que reconozca las realidades de nuestros países y también dé respuestas eficientes a sus necesidades y problemas. Es necesario para la región superar nuestra tendencia a las malas copias de modelos foráneos a nuestras realidades, los cuales no han podido responder a las exigencias de nuestros pueblos.

A veces creemos que lo que se aplica en Europa o Estados Unidos o en cualquier lugar del primer mundo en torno a la democracia se puede aplicar a nuestros países, el problema es no integrar nuestras visiones y pensamientos en términos de igualdad y horizontalidad a la construcción de nuestros sistemas políticos. Por lo tanto, la construcción de esa democracia integradora, solidaria y deliberativa es una tarea colectiva y plural entre todas y todos.

Nuestros países requieren de una ciudadanía responsable de lo que decide y al mismo tiempo consciente de sus roles históricos, pero eso no viene únicamente por las condiciones sociales, políticas y culturales, esto llega cuando somos capaces de sentir y de actuar como agentes de cambio, desde el lugar, el ámbito o el espacio en el que estemos.

En este sentido, el reto es empezar a mirar desde el sur y para el sur, esto adquiere un análisis necesario, cuando pensamos en la construcción de América Latina y el Caribe como región integrada, eliminando las 


\section{DERECHO ELECTORAL}

fronteras entre nuestros pueblos y materializando ese bienestar colectivo que anhelamos.

En cuanto al segundo eje de fortalecimiento de la institucionalidad democrática, los países latinoamericanos y caribeños deben realizar reformas que robustezcan el ordenamiento jurídico institucional para dar cabida a las demandas sociales de igualdad y participación. En este sentido, el diálogo plural y los enlaces entre actores políticos, ciudadanía y Estado son imprescindibles para fijar el contenido, alcances y objetivos de toda reforma que promueva la ampliación y garantía de los derechos políticos, la participación de las organizaciones políticas, la organización de elecciones, la representatividad política y otros factores que configuran el sistema electoral de cada país y que contribuyen al fortalecimiento de la gobernabilidad democrática. Sin este diálogo y consenso ampliado, las reformas fracasan y son de fugaz existencia y vigencia y se vuelven coyunturales y carentes de legitimidad democrática.

En este sentido, los parlamentos nacionales y sus legisladores siempre deben tener en cuenta que todo afán de cambio, enmienda o reforma constitucional o legal debe generar un paso adelante o evolución del sistema democrático y sus componentes, donde es muy importante la coherencia dentro de la norma, evitar o reducir las contradicciones que dificulten su aplicación, delimitar claramente el rol de los actores políticos y sociales y de los organismos electorales. Es decir, la norma electoral debe guardar orden, integralidad, coherencia e insertarse de forma armónica en el marco constitucional y, sobre todo, responder al interés general.

El tercer eje es el diseño de políticas públicas con real y efectiva participación ciudadana a fin de que acompañen los avances normativos, en que resulta indispensable y medular que todos los procesos impulsados y aprobados mediante mecanismos de democracia directa como referéndums y consultas populares sean interiorizados y apropiados por la ciudadanía, de manera que promuevan una participación ciudadana empoderada y comprometida con sus derechos y con la democracia.

En este contexto, desde las instituciones debe cambiar la concepción tradicional de las políticas públicas, en la cual únicamente contamos con la participación ciudadana en la etapa de evaluación. El reto es que la participación ciudadana esté presente en los 4 ciclos de construcción de las políticas públicas: en el diagnóstico, en la formulación, en la ejecución 


\section{DERECHO ELECTORAL}

y en la evaluación. Solo así podremos generar políticas que tengan impacto, caso contrario, no tendremos políticas públicas que empoderen a los ciudadanos y tengan la real incidencia que buscamos y necesitamos.

No podemos tener instituciones que no abran la puerta a la participación ciudadana o que sean apáticas y lejanas a los ciudadanos. Es imposible que se puedan alcanzar los objetivos desde un escritorio; los cambios se dan cuando se trabaja de forma descentralizada, junto con la gente en sus espacios territoriales, entendiendo sus necesidades y abriendo canales reales de participación.

Nos queda como apuesta al futuro inmediato el «trabajo de ciudadanización» de las organizaciones políticas, las cuales deben incorporar, impostergablemente, el cumplimiento total de la equidad de género, la paridad vertical y horizontal en sus cuerpos colegiados, la diversidad étnica y la representación geográfica de la urbanidad y la ruralidad en sus directivas. Integrando en su acción política mecanismos reales de democracia interna como primarias abiertas, cerradas 0 asambleas; el reto es democratizar las organizaciones políticas y que se conviertan en puentes efectivos y transparentes entre el ejercicio del poder y la ciudadanía. De esta manera, las organizaciones políticas tendrán candidatos que surjan del apoyo y consenso de sus integrantes, y no de la voluntad de gerentes-propietarios o dueños de los partidos políticos que los designan a través de negociaciones de pasillo o con el apoyo de grandes chequeras. De forma paralela a esto, es necesario entender el real contenido de la democracia comunitaria y el ejercicio de los derechos colectivos y profundizar en su reconocimiento y aplicación como complemento a las democracias representativa y directa, las cuales están reconocidas en las constituciones de Bolivia y Ecuador.

La democracia es más que una cuestión procedimental, debe incorporar las diversas visiones sociales y territoriales, desde la unidad geográfica más pequeña hasta la más grande; integrando a todos los territorios, a todas las personas y pueblos desde una visión nacional que transcienda a lo local, regional y comunitario.

Finalmente, el cuarto eje referente a la incorporación del enfoque de protección de derechos humanos en los ámbitos políticos-electorales, tanto los parlamentos nacionales como los organismos electorales, los partidos políticos y movimientos sociales, al momento de proponer y generar una propuesta de reforma para aplicarla a un contexto social 


\section{DERECHO EIECTORAL}

determinado, deben partir de un criterio amplio sobre las fuentes que sustentan la construcción de la norma. El marco constitucional debe ser el primer instrumento de referencia para determinar el marco y contenido de dicha reforma; sin embargo, no solo se agota en este, en la actualidad la jurisprudencia de las cortes y tribunales constitucionales de la región constituyen una fuente indispensable para generar avances sustantivos y proveer elementos que contribuyen al avance del ordenamiento jurídicoelectoral.

También, los instrumentos internacionales de derechos humanos, específicamente aquellos celebrados en el marco del sistema interamericano de derechos humanos, así como las opiniones consultivas de la Comisión y las sentencias de la Corte Interamericana, las mismas que establecen parámetros normativos y jurisprudenciales transcendentales para el mejoramiento $y$ el avance normativo e institucional en materia electoral.

Todos estos instrumentos generan seguridad jurídica al obtener una conducta previsible de las autoridades electorales, la eliminación de arbitrariedades en el ejercicio de las funciones de los organismos electorales, la estabilidad y certeza en el desenvolvimiento del proceso electoral, tanto para los ciudadanos como para los candidatos y organizaciones políticas, con lo que se logra superar las oposiciones y solucionar las controversias.

\section{Colofón: ROBUSTECER LA DEMOCRACIA FRENTE A LOS NUBARRONES DEL PRESENTE}

Definitivamente, en esta segunda década del siglo XXI, en la que estamos viviendo tiempos de pandemia (covid-19), se levantan tiempos y desafíos complejos para el mundo y la región; para todos los Estados, ciudadanos e instituciones. El balance nos presenta aún una democracia formal, de carácter eminentemente electoral, en donde todavía encontramos una desigualdad lacerante, injusticia, pobreza y la exclusión de las grandes mayorías; sobre la cual se ciernen los devaneos totalitarios propios e importados a la región.

El mayor peligro para una democracia es tener ciudadanos ausentes; frente a esto y de cara al futuro necesitamos promover los espacios de 


\section{DERECHO EIECTORAL}

participación ciudadana, ciudadanizar la política y potencializar todos nuestros esfuerzos para fortalecer la democracia.

No podemos olvidarnos de que el centro de todo sistema democrático, económico y productivo es el ser humano, que es el principio y el fin de todo. Por eso, nuestros esfuerzos deben estar orientados al fortalecimiento de sociedades humanistas, democráticas, con conciencia social; a la búsqueda de sociedades diversas e incluyentes, en donde exista espacio para todas las voces, garantizando el ejercicio pleno de las libertades.

Así, en todo tiempo, el único camino posible y la apuesta de todos los Estados y ciudadanos en la región es perfeccionar, robustecer, defender y ampliar la democracia, las elecciones y los derechos humanos, y lograr esa inclusión, justicia, bienestar y solidaridad para la gente de nuestros países, como lo dijo en 1979 el expresidente del Ecuador, Jaime Roldós Aguilera (1979-1981) en su discurso de posesión "(...) La democracia no es perfecta, pero es susceptible de mejoramiento. (...). Solo dentro de una democracia vigorosa, cimentada en la solidaridad, la justicia y el bienestar real de nuestros pueblos puede construirse un futuro brillante frente a los nubarrones del presente" (Roldós, 1979).

En la nueva realidad mundial que estamos viviendo al empezar el 2020, solo hay espacio para la cooperación, la solidaridad, la empatía y el trabajo. La salida de la crisis debe implicar el compromiso regional y mundial de no dejar a nadie atrás.

\section{REFERENCIAS BIBLIOGRÁFICAS}

Allende, S. (27 agosto, 1971). Cada pueblo: su propia ruta. Discurso pronunciado en la Municipalidad de Guayaquil, Ecuador. Recuperado de https://www.marxists.org/espanol/allende/1971/agosto27.htm

Bitar, S. (14 enero, 2020). Los desafíos a la democracia en América Latina. News IDEA Internacional. Recuperado de: https://www.idea.int/es/newsmedia/news/es/los-desaf\%C3\%ADos-la-democracia-en-am\%C3\%A9ricalatina 


\section{DERECHO EIECTORAL}

Comisión Económica para América Latina (CEPAL) de la Organización de las Naciones Unidas (2019). Panorama Social de América Latina. Recuperado de https://repositorio.cepal.org/bitstream/handle/11362/44969 /5/S1901133_es.pdf

Corporación Latinobarómetro (2018). Informe 2018. Recuperado de www.latinobarometro.org

Couso, J. (2011). Los desafíos de la democracia constitucional en América Latina: entre la tentación populista y la utopía neoconstitucionales. Recuperado de https://core.ac.uk/download/pdf/46536238.pdf

Freidenberg, F. (2014). Las Elecciones en América Latina (2013-2014). Revista Mexicana de Derecho Electoral, (7-8), 267-294 Recuperado de https://revistas.juridicas.unam.mx/index.php/derechoelectoral/article/view/10092

Guerrero, F. (29 agosto, 2019). Otoño turbulento. Excelsior. Recuperado de https://m.excelsior.com.mx/opinion/francisco-guerreroaguirre/otonoturbulento/1344569

Guerrero, F. (2020). Democracia en tiempos de pandemia. Manuscrito presentado para publicación.

IDEA Internacional (23 de marzo, 2020). Panorama global del impacto del COVID-19 en las elecciones. Recuperado de https://www.idea.int/es/newsmedia/multimedia-reports/panorama-global-del-impacto-del-covid-19-enlas-elecciones

Nohlen, D. (2003). Desafíos de la Democracia Contemporánea. Justicia Electoral, (18), 39-44. Recuperado de https://revistascolaboracion.juridicas.unam. $\mathrm{mx} /$ index.php/justicia-electoral/article/viewFile /11974/10781

Roldós, J. (10 agosto, 1979). Discurso de posesión. Recuperado de https://www.youtube.com/watch?v=eLoojD-4TPE 